ARTICLE

https://doi.org/10.1038/s41467-019-09807-5 OPEN

\title{
Transient opening of trimeric prefusion RSV F proteins
}

Morgan S.A. Gilman 1,2, Polina Furmanova-Hollenstein'3 , Gabriel Pascual' ${ }^{4}$, Angélique B. van 't Wout (1) ${ }^{5,6}$, Johannes P.M. Langedijk ${ }^{3} \&$ Jason S. McLellan (i) ${ }^{2}$

The respiratory syncytial virus (RSV) F glycoprotein is a class I fusion protein that mediates viral entry and is a major target of neutralizing antibodies. Structures of prefusion forms of RSV F, as well as other class I fusion proteins, have revealed compact trimeric arrangements, yet whether these trimeric forms can transiently open remains unknown. Here, we perform structural and biochemical studies on a recently isolated antibody, CR9501, and demonstrate that it enhances the opening of prefusion-stabilized RSV F trimers. The $3.3 \AA$ crystal structure of monomeric RSV F bound to CR9501, combined with analysis of over 25 previously determined RSV F structures, reveals a breathing motion of the prefusion conformation. We also demonstrate that full-length RSV F trimers transiently open and dissociate on the cell surface. Collectively, these findings have implications for the function of class I fusion proteins, as well as antibody prophylaxis and vaccine development for RSV.

\footnotetext{
${ }^{1}$ Department of Biochemistry and Cell Biology, Geisel School of Medicine at Dartmouth, Hanover, NH 03755, USA. ${ }^{2}$ Department of Molecular Biosciences, University of Texas at Austin, Austin, TX 78712, USA. ${ }^{3}$ Janssen Infectious Diseases and Vaccines, Leiden, CN 2333, The Netherlands. ${ }^{4}$ Janssen Immunosciences, World Without Disease Accelerator, San Diego, CA 92121, USA. ${ }^{5}$ Janssen Prevention Center, Janssen Vaccines \& Prevention B.V, Leiden, CN 2333, The Netherlands. ${ }^{6}$ Present address: AlphaBiomics, London SW4 OPA, United Kingdom. Correspondence and requests for materials should be addressed to J.P.M.L. (email: hlangedi@its.jnj.com) or to J.S.M. (email: jmclellan@austin.utexas.edu)
} 
$\mathrm{R}$ espiratory syncytial virus (RSV) is an enveloped RNA virus that is a member of the recently established Pneumoviridae family ${ }^{1}$. Upper respiratory tract infections due to RSV reoccur multiple times throughout life, but rarely lead to severe complications in healthy adults. However, RSV infections in infants, the elderly, and the immunocompromised can lead to bronchiolitis or pneumonia, which may result in hospitalization or even death. These complications are a substantial cause of infant mortality worldwide, resulting in approximately 60,000 inhospital deaths annually in children under the age of $5^{2}$. Although mortality due to RSV infections is rare in developed countries, the economic burden associated with RSV is substantial. In the United States, for example, it is estimated that 652 million dollars were spent in 2000 alone on RSV-related medical costs ${ }^{3}$. Although prophylaxis with the monoclonal antibody palivizumab reduces the risk of hospitalization associated with RSV, it must be delivered intravenously multiple times per RSV season and has modest efficacy, preventing its use in developing regions $s^{4,5}$.

Two glycoproteins are present on the surface of RSV-the attachment protein (RSV G) and the fusion protein (RSV F). RSV $G$ enhances pathogenicity in vivo and facilitates attachment to host cells ${ }^{6}$, but is not absolutely required for infection ${ }^{7,8}$. RSV F is a class I fusion protein that mediates fusion of the viral membrane with a host-cell membrane, a process that poses an otherwise insurmountable energetic barrier to viral entry. Initially, the $\mathrm{F}$ protein exists in a metastable prefusion conformation that is anchored in the viral membrane by a transmembrane domain. In this conformation the hydrophobic fusion peptide is buried within the central cavity of the protein ${ }^{9}$. An unknown event causes prefusion $\mathrm{F}$ to undergo a dramatic conformational change, resulting in refolding of five distinct structural elements adjacent to the fusion peptide into a single alpha helix and insertion of the fusion peptide into the host-cell membrane. In this state, called the pre-hairpin intermediate, two heptad repeats (HRA and HRB) are positioned on opposing ends of the F molecule. The collapse of these heptad repeats into a stable sixhelix bundle is an energetically favorable conformational change that is coupled to the fusion of the two lipid bilayers ${ }^{10,11}$. Antibodies that bind to $\mathrm{F}$ can interrupt the fusion process, thereby preventing viral entry and reducing the severity of RSV-related disease $5,12,13$. Thus, there is great interest in the isolation of RSV F-directed antibodies with neutralizing activity that could serve as the basis for improved therapeutics.

Mapping the antigenic topology of RSV F previously relied upon competition assays and isolation of viral escape mutations $^{14-17}$, but recent efforts have expanded upon these maps and placed them in the context of the high-resolution structures for both pre- and postfusion $\mathrm{F}^{9-11,18}$. Among the first antigenic sites to be defined was antigenic site II, which is composed of a helixturn-helix motif that spans residues 253-278 and is the target of palivizumab ${ }^{19-21}$. This motif is present on both the pre- and postfusion conformations of $\mathrm{F}$, and antibodies that recognize site II generally bind equally well to both ${ }^{9,22}$. Recently, it has been established that antibodies that specifically recognize the prefusion conformation of $\mathrm{F}$ are in general more potently neutralizing than antibodies that also bind to postfusion $\mathrm{F}^{23}$. The first prefusion-specific antibodies that were isolated recognized the apex of the prefusion F trimer and neutralized RSV over tenfold more potently than palivizumab 9,24 . This region was named antigenic site $\varnothing$ and was later shown to be a primary target of RSV-neutralizing activity in human sera ${ }^{25}$. A second prefusionspecific epitope, which we call site $V$, is composed of $\alpha 2-\alpha 3$ and $\beta 3-\beta 4^{23,26}$. These secondary structure elements all refold into the HRA that forms the center of the six-helix bundle in postfusion $F$ and therefore these antibodies are likely to exhibit the highest specificity for prefusion $\mathrm{F}$.
Like many other class I fusion proteins, RSV F undergoes proteolytic processing during maturation in the secretory pathway of infected cells. RSV F is synthesized as a single-chain inactive precursor called $F_{0}$ that contains three subunits: $F_{1}, F_{2}$, and a 27 -amino acid glycopeptide called pep $27^{27}$. This precursor must be cleaved by a furin-like protease to release pep27 and form the mature, fusion-competent protein ${ }^{27-30}$. The C-terminal $\mathrm{F}_{1}$ subunit contains the transmembrane domain, two heptad repeats, and an $\mathrm{N}$-terminal fusion peptide. Residues in the $\mathrm{F}_{2}$ subunit contribute to fusogenicity of the $\mathrm{F}$ protein and possibly the species specificity of RSV ${ }^{31-35}$. In the mature protein, the $F_{1}$ and $\mathrm{F}_{2}$ subunits are covalently associated via two disulfide bonds ${ }^{10,11,30}$. Three $F_{1}-F_{2}$ protomers then associate via weak intermolecular interactions to form the trimeric, prefusion protein on the surface of the virion. However, these interactions are not sufficient to drive trimerization of the prefusion RSV F ectodomain and therefore engineering of soluble trimeric prefusion $\mathrm{F}$ proteins has required the inclusion of artificial trimerization motifs 9,36

For class I fusion proteins that lack a fusion-suppressive capping domain, such as the $\mathrm{F}$ proteins from pneumoviruses and the related paramyxoviruses $9,37,38$, the extent to which they remain as closed trimers in the context of biological membranes remains unknown. Here, we investigate the dynamic nature of RSV F trimers in solution and on membranes due to the serendipitous discovery of a monoclonal antibody with unique properties. We find that antibody CR9501 favors the opening of soluble, prefusion $\mathrm{F}$ trimers and exhibits distinct competition profiles on trimeric and monomeric prefusion $\mathrm{F}$ proteins. Analysis of multiple prefusion $\mathrm{F}$ structures identifies two conformational states that are related by a breathing motion. We utilize the unique properties of CR9501 to demonstrate that the prefusion RSV F trimer is in a monomer-trimer equilibrium when expressed on the surface of mammalian cells. These findings suggest that prefusion RSV F trimers are dynamic molecules capable of transiently opening and dissociating, a discovery that has implications for the structure and function of class I fusion proteins as well as RSV vaccine development.

\section{Results}

CR9501 potently neutralizes RSV and binds antigenic site $\mathrm{V}$. Antibody CR9501 was isolated from the peripheral blood mononuclear cells (PBMCs) of a human donor and found to potently neutralize RSV (Fig. 1a). CR9501 neutralized a small panel of subtype $A$ and $B$ viruses with half-maximal inhibitory concentration $\left(\mathrm{IC}_{50}\right)$ values ranging from 0.06 to $0.23 \mathrm{nM}$, approximately 7-40-fold more potently than palivizumab. The IC $_{50}$ of CR9501 was similar to that of the prefusion F-specific antibody D25 for three of the four viruses tested. Initial characterization by shotgun mutagenesis identified an epitope that includes site $\mathrm{V}$ and portions of the $\mathrm{F}_{2}$ subunit (Fig. $1 \mathrm{~b}$ and Supplementary Table 1), which are in close proximity in the prefusion F structure but are separated by more than $90 \AA$ in postfusion $F$, suggesting that CR9501 may be prefusion F-specific. Consistent with this result, CR9501 showed undetectable levels of binding to postfusion RSV F as measured by surface plasmon resonance (SPR) (Supplementary Fig. 1).

To better define the epitope, we attempted to crystallize CR9501 in complex with a prefusion-stabilized F ectodomain that contained a C-terminal Foldon trimerization motif (a construct referred to as $\mathrm{PR}-\mathrm{DM}^{33}$ ). Because crystallization efforts of this binary complex yielded poorly diffracting crystals, we attempted to generate a ternary complex of prefusion F with CR9501 and motavizumab, an antibody against antigenic site II. Simultaneous addition of CR9501 and motavizumab Fabs resulted in the 
a

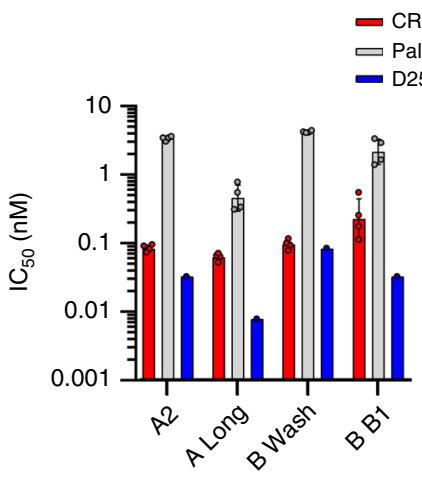

b

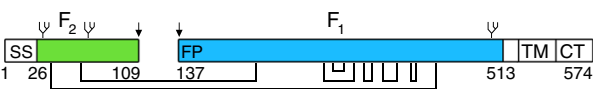

CR9501 Palivizumab D25
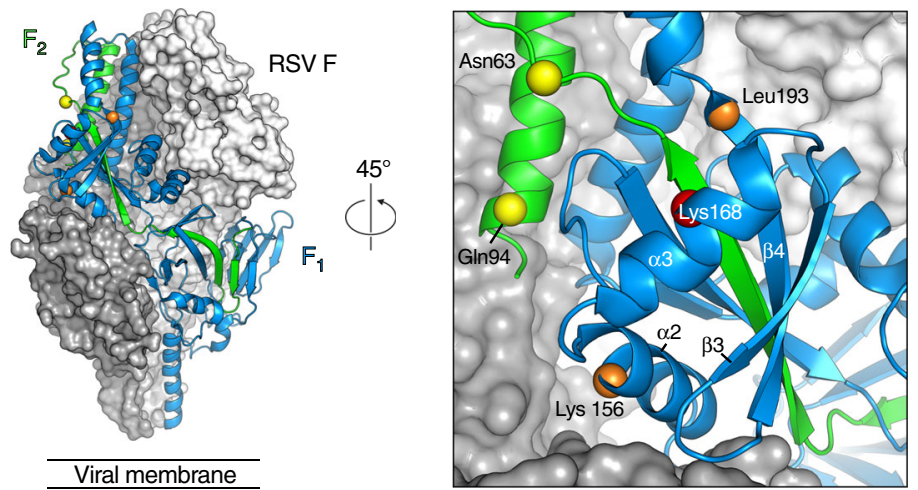

Fig. 1 CR9501 potently neutralizes RSV and binds near antigenic site V. a Geometric mean neutralization IC 50 values for CR9501 (red), palivizumab (gray), and D25 (blue). Error bars denote standard deviation of geometric means ( $n=4$ biologically independent experiments with nine technical replicates for CR9501 and palivizumab and $n=1$ biologically independent experiment with 27-43 technical replicates for D25). $\mathbf{b}$ Two protomers of prefusion $\mathrm{F}$ are shown as molecular surfaces colored gray and white. The third protomer is shown as ribbons, with the $F_{1}$ subunit colored blue and the $F_{2}$ subunit colored green. The $\mathrm{C} \alpha$ atoms of residues identified by shotgun mutagenesis are shown as spheres and colored according to Supplementary Table 1. The inset shows a zoomed view of a $45^{\circ}$ rotation about the trimeric axis. A linear depiction of $F$ is shown at the top right, with the $F_{1}$ and $F_{2}$ subunits colored blue and green, respectively. Regions colored white are absent in the soluble protein (SS signal sequence, TM transmembrane, CT cytoplasmic tail). Glycans are shown as branches, disulfide bonds are shown as black lines, and furin cleavage sites are indicated by black arrows

formation of two complexes with distinct elution volumes on size-exclusion chromatography (Supplementary Fig. 2, black trace). The earlier-eluting peak contained the ternary complex, whereas the later-eluting peak contained RSV F bound only to one Fab. This suggested that there was an order-dependent competition between the antibodies, where initial binding of one antibody prevented binding of the other. To test this hypothesis, we incubated prefusion $\mathrm{F}$ with CR9501 prior to the addition of motavizumab. This resulted in a single peak on size-exclusion chromatography with an elution volume corresponding to that of the ternary complex (Supplementary Fig. 2, red trace). In contrast, preincubating prefusion $\mathrm{F}$ with motavizumab prior to addition of CR9501 resulted in a decrease of the ternary complex fraction and a corresponding increase in binary complex (Supplementary Fig. 2, blue trace). This order-dependent binding could be due to an antibody-induced or antibody-selected conformational change of RSV F.

CR9501 and motavizumab exhibit interprotomeric competition. We had previously predicted that interprotomeric competition may occur between antibodies that recognize antigenic sites II and $\mathrm{V}^{23}$. We therefore tested the competition of CR9501 and motavizumab on trimeric and monomeric prefusion $\mathrm{F}$ proteins using SPR (Fig. 2). Two previously described variants of trimeric prefusion F (PR-DM and DS-Cav1) were included in these studies. These variants each contain a Foldon trimerization motif but have different stabilizing substitutions. The DS-Cav1 variant contains an additional disulfide bond (S155C/S290C) as well as two cavity-filling mutations (S190F/V207L), whereas PR-DM contains one proline substitution $(\mathrm{S} 215 \mathrm{P})$ and one mutation in the $\mathrm{F}_{2}$ subunit $(\mathrm{N} 67 \mathrm{I})$ and is the more stable of the two variants $^{33,39}$. The monomeric prefusion $\mathrm{F}$ protein contains the DS-Cav1 stabilizing substitutions but lacks the trimerization domain and elutes at a volume consistent with a single prefusion F protomer on size-exclusion chromatography (Supplementary Fig. 3). For these experiments, the RSV F variants were immobilized on the chip and a sequential injection of CR9501 Fab followed by motavizumab Fab, or motavizumab Fab followed by
CR9501 Fab, was performed. For trimeric, prefusion-stabilized variants, saturating the $\mathrm{F}$ protein with CR9501 Fab allowed binding of motavizumab Fab (Fig. 2a, b, top panel, red trace), although the response was lower than observed for binding to unliganded prefusion F (Fig. 2a, b, bottom panel, red trace). When trimeric prefusion $F$ proteins were first saturated with motavizumab, little-to-no additional binding was observed upon injection of CR9501 Fab (Fig. 2a, b, top panel, black trace). In contrast, CR9501 and motavizumab did not compete for binding to monomeric prefusion F, as expected (Fig. 2c, top and bottom panels). To verify that this competition profile with CR9501 was unique to antibodies that recognize site II, competition between CR9501 and the site $\varnothing$-specific antibody D25 was also measured. Antibodies against site $\varnothing$ are expected to partially overlap with site $\mathrm{V}$ within a single protomer and therefore competition between antibodies recognizing sites $\varnothing$ and $V$ would not be expected to depend on trimerization of prefusion F. Consistent with this result, D25 competed with CR9501 on both trimeric and monomeric prefusion F (Supplementary Fig. 4). Collectively, these results indicate that CR9501 and motavizumab competition is interprotomeric and suggest that CR9501 binding reduces their competition by favoring opening of the prefusion $\mathrm{F}$ trimer.

Based on the results of the competition analysis, we proposed the following model (illustrated in Fig. 2d): (1) the prefusion F trimer exists in an equilibrium between an open and closed state, but the Foldon trimerization motif tethers the protomers and prevents complete dissociation, (2) CR9501 binding favors trimer opening, (3) opening of the CR9501-bound trimer allows motavizumab to bind by relieving the steric hindrance across neighboring protomers, (4) once the ternary complex has formed, the clash between motavizumab and CR9501 prevents the collapse of the complex back to the closed trimeric state, (5) because both Fabs have essentially no off-rate, the equilibrium is driven to a final state comprising each protomer bound to both Fabs.

Closer inspection of the binding rates for the two prefusion $\mathrm{F}$ proteins reveals a number of differences that provide insights about the propensity of these two variants to sample the open conformation. First, the rate of CR9501 association with 

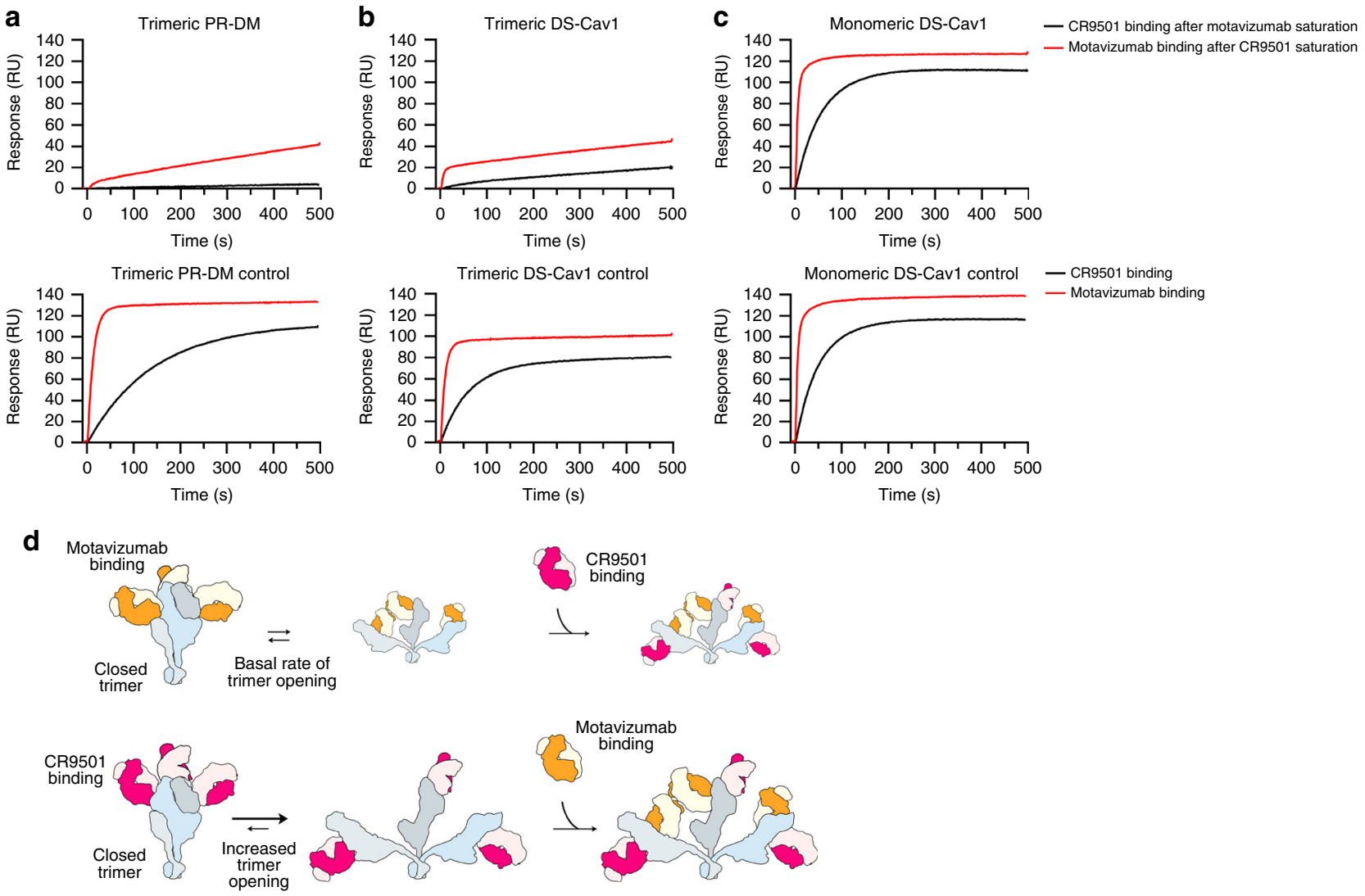

Fig. 2 CR9501 enhances opening of prefusion F. Two prefusion F variants that include previously described stabilizing mutations and a trimerization motifa PR-DM or b DS-Cav1-or $\mathbf{c}$ a prefusion-stabilized variant lacking the trimerization motif (monomeric DS-Cav1) were immobilized and bound with saturating levels of either motavizumab or CR9501. A second Fab, either CR9501 (black) or motavizumab (red), was then injected over the surface while measuring the binding response. The bottom row shows the controls in which buffer replaced the first Fab incubation. $\mathbf{d}$ Model for binding of CR9501 and motavizumab to soluble prefusion $\mathrm{F}$ protein illustrating the effects of antibody order-of-addition on trimer opening. The cartoon depicts prefusion $\mathrm{F}$ protomers in blue, light gray, and dark gray, CR9501 in pink and light pink, and motavizumab in orange and tan

motavizumab-saturated PR-DM, which should reflect the natural rate of PR-DM opening, is very low (Fig. 2a, top panel, black curve). Second, the rate of CR9501 association with motavizumab-saturated DS-Cav1 is higher than observed for PR-DM (Fig. 2b, top panel, black curve), suggesting that DS-Cav1 naturally samples an open state more frequently than does PRDM. Third, the rate of motavizumab association with CR9501saturated PR-DM correlates with the CR9501-induced rate of trimer opening because the basal rate of PR-DM opening in the absence of antibodies is negligible (Fig. 2a, top panel, red curve). Finally, two different association rates are visible in the association of motavizumab with CR9501-saturated DS-Cav1 (Fig. 2b, top panel, red curve). The first rate likely represents the fraction of DS-Cav1 that is already present in an open conformation and can readily bind to motavizumab upon injection. This rate is very fast, similar to the rate of motavizumab binding to unliganded DS-Cav1 (Fig. 2b, bottom panel, red curve). The second, slower rate correlates with the rate of DSCavl opening and is only slightly increased in the presence of CR9501, again suggesting that DS-Cav1 samples an open conformation more often than PR-DM. Consistent with this hypothesis, DS-Cav1 incubated with motavizumab prior to addition of CR9501 resulted in almost entirely ternary complex as measured by SEC (Supplementary Fig. 2, blue trace).

Structure reveals interprotomeric clash between antibodies. To avoid conformational heterogeneity resulting from CR9501induced opening of prefusion $\mathrm{F}$ trimers, we sought to determine a CR9501-bound F structure by using the monomeric variant of prefusion $\mathrm{F}$. This complex formed crystals in space group $P 2_{1} 2_{1} 2_{1}$ that diffracted X-rays to $3.3 \AA$ resolution (Supplementary Table 2). The structure revealed that CR9501 recognizes antigenic site $\mathrm{V}$, but also makes substantial contacts with the $\mathrm{F}_{2}$ subunit (Fig. 3a), consistent with the results of shotgun mutagenesis. The third heavy chain complementaritydetermining region (CDR H3) of CR9501 is inserted between two elements of the $\mathrm{F}_{2}$ subunit-the al helix and the loop that connects this helix to $\beta 2$ (Fig. 3b). CR9501 uses both the heavy and light chain CDRs to contact five discrete regions of the linear RSV F protein sequence that are in close proximity in the prefusion $\mathrm{F}$ conformation (Fig. 3c). The light chain interacts with four of these regions, forming a salt bridge with Lys65 and a hydrogen bond with Glu66, both of which are on the loop connecting $\beta 2$ and $\alpha 1$ in the $F_{2}$ subunit. In addition, the light chain forms hydrogen bonds with two residues in the $\alpha 3$ helix (Asn165 and Lys168) and on the loop connecting $\beta 5$ and $\beta 6$ (Glu294 and Glu295). The heavy chain makes fewer contacts, the majority of which are with the $\mathrm{F}_{2}$ subunit. The disulfide-stabilized CDR H3 forms hydrogen bonds with Asn63 and Asp84 and a salt bridge with Lys65. A salt bridge is also formed between CDR H1 and Lys 87 of the $\alpha 1$ helix. In the postfusion $F$ conformation, $a 3$ would be separated from the other elements of the CR9501 epitope by more than $90 \AA$, suggesting that CR9501 neutralizes RSV by trapping the prefusion conformation of $\mathrm{F}$ - acting as a staple that links the a3 helix to more conformationally stable regions of the prefusion F protein. 
a

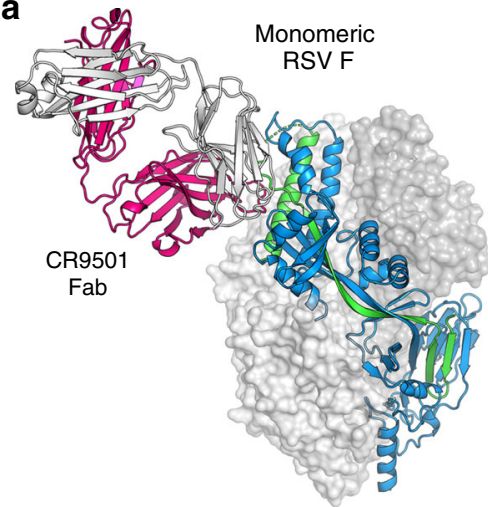

b

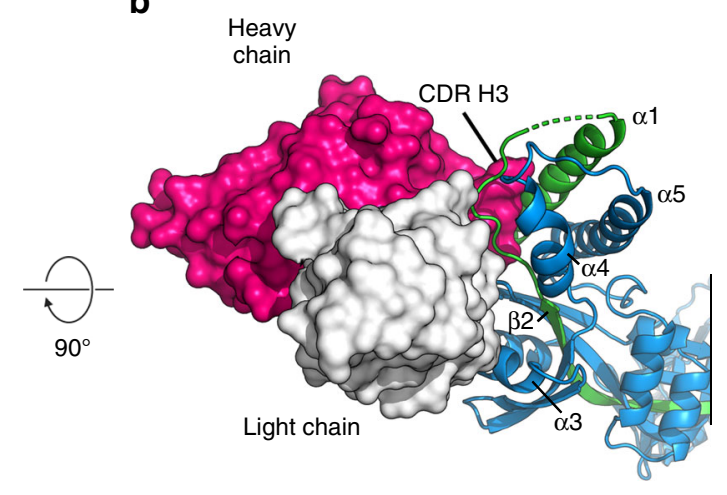

Viral membrane

C
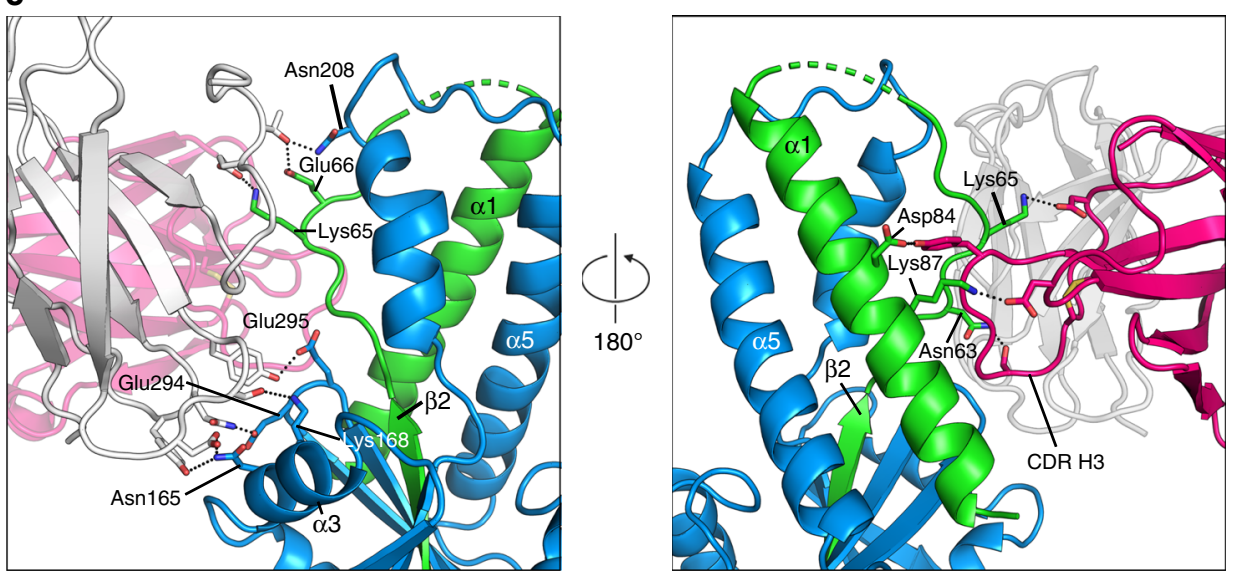

Fig. 3 Crystal structure of CR9501 bound to monomeric prefusion F. a Monomeric prefusion F is shown as ribbons, with the $F_{1}$ subunit colored blue and the $F_{2}$ subunit colored green. The CR9501 heavy and light chains are shown as pink and white ribbons, respectively. The two additional protomers of the prefusion $\mathrm{F}$ trimer are generated using the $\mathrm{C} 3$ symmetry observed in previous crystal structures and shown as transparent molecular surfaces. $\mathbf{b} \mathrm{A} 90^{\circ}$ rotation about the horizontal axis with the CR9501 variable domain shown as molecular surfaces illustrates the insertion of the CDR H3 between two regions of the $\mathrm{F}_{2}$ subunit. c $\mathrm{A}$ zoomed-in view of the orientation in (a) (left) and a $180^{\circ}$ rotation about the trimeric axis (right). Side chains involved in hydrogen bonds or salt bridges are shown as sticks with oxygen and nitrogen atoms colored red and blue, respectively. Hydrogen bonds and salt bridges are depicted as black dotted lines

We generated the biological RSV F trimer by applying the threefold symmetry observed in previous structures to the monomeric complex. This model revealed that the CR9501 Fab bound to one RSV F protomer would clash substantially with the motavizumab Fab bound to the neighboring protomer (Fig. 4). This indicates that in order for the ternary complex to form, the prefusion $\mathrm{F}$ trimer must be in an open conformation that alleviates the interprotomeric clash between these two antibodies (Supplementary Movie 1), as predicted based on the results of the competition analysis.

CR9501 causes prefusion F trimers to splay open in solution. To validate the model proposed above, we sought to capture the prefusion $\mathrm{F}$ trimer in the splayed-open state. We therefore preincubated PR-DM with CR9501 before addition of motavizumab to drive the equilibrium toward the fully open conformation (Fig. 5a). This complex was analyzed by negative-stain EM, but because the orientation of each protomer relative to the other protomers in a splayed-open trimer is variable, $2 \mathrm{D}$ classification did not yield a splayed-open trimer but rather a single protomer bound by two Fabs (Supplementary Fig. 5). Therefore, we crystallized the complex, in hopes of capturing a static view of a splayed-open trimer. The complex formed crystals in space group
$P 6_{1}$ that diffracted X-rays to $4.1 \AA$ resolution (Supplementary Table 2). Molecular replacement identified one RSV F protomer bound to one CR9501 Fab and one motavizumab Fab in the asymmetric unit. Because $P 6_{1}$ lacks a non-screw threefold axis, a biological trimer could not be generated by symmetry operations, indicating that the protomers dissociated during (or prior to) crystallization (Fig. 5b, c). Although the density for the Cterminal portion of RSV F is not visible, the orientation of each monomer in the unit cell is compatible with the distance required for Foldon to remain trimeric while also allowing the dissociated protomers to pack along the sixfold screw axis (Fig. 5c). Thus, our crystallographic analysis again suggests the presence of a splayedopen trimer.

Because crystallization can select for conformations that do not predominate in solution, we sought to estimate the extent to which CR9501 binding alters the monomer-trimer equilibrium using another method. We performed negative-stain EM on CR9501-bound prefusion F (PR-DM) and compared it to the negative-stain EM for motavizumab-bound prefusion F (Fig. 5d, e). Micrographs for the prefusion F-motavizumab complex were primarily composed of top-down views of the trimer bound by three Fabs (Fig. 5d). In contrast, the micrographs of the prefusion F-CR9501 complex contained three distinct particle classes in which the CR9501-bound trimer resembled previously 
a

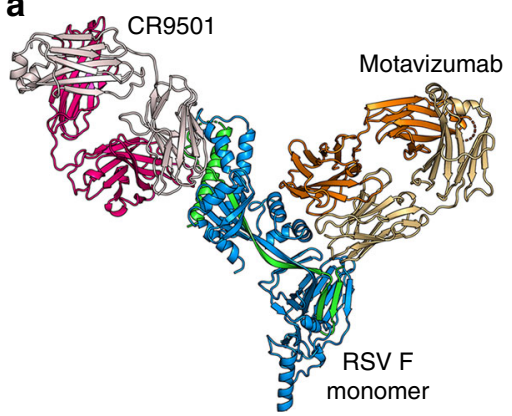

C

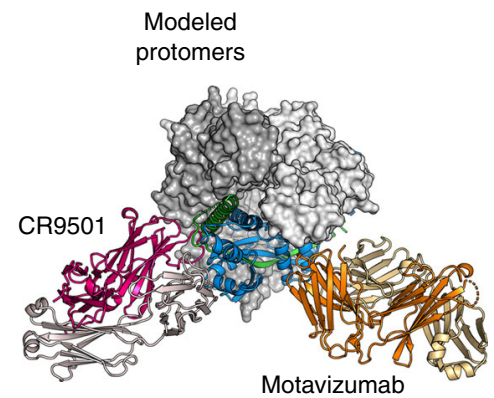

b

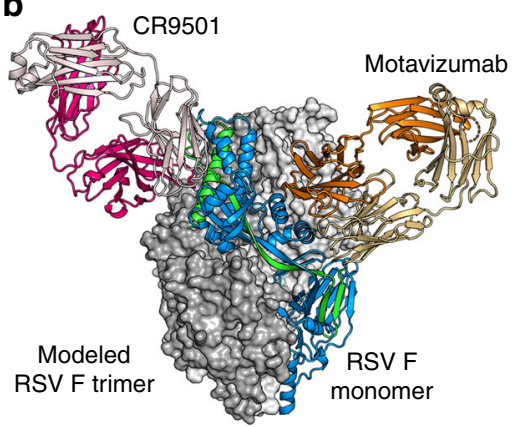

d

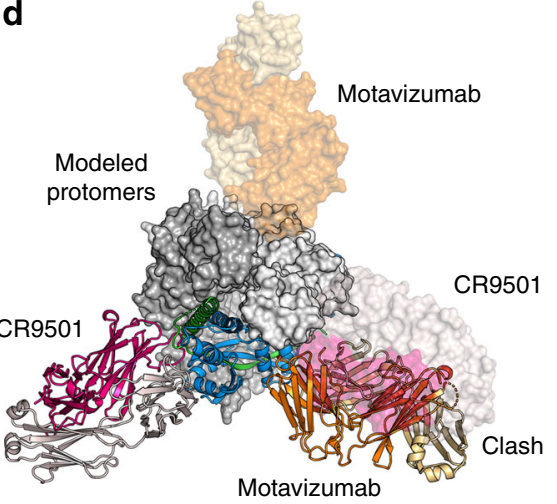

Fig. 4 CR9501 and motavizumab Fabs cannot simultaneously bind to closed, trimeric F. a Monomeric prefusion F-CR9501 structure is shown in ribbons with the $F_{1}$ subunit colored blue and the $F_{2}$ subunit colored green. The CR9501 heavy and light chains are shown as dark pink and light pink ribbons, respectively. Motavizumab Fab is modeled onto the prefusion $\mathrm{F}$ monomer using the previously solved structure (PDB ID:3IXT) and is shown in ribbons with the heavy and light chains colored orange and tan, respectively. $\mathbf{b}$ The two additional protomers of the prefusion $\mathrm{F}$ trimer were generated using the C3 symmetry observed in previous crystal structures and are shown as molecular surfaces. c A $90^{\circ}$ rotation about the horizontal axis of the complex shown in (b), viewed looking toward the viral membrane. d A second asymmetric unit from the crystal structure, composed of one prefusion $\mathrm{F}$ protomer and one CR9501 Fab, and one modeled motavizumab Fab were aligned with a neighboring protomer of the biological trimer and are shown as transparent molecular surfaces. An interprotomeric clash is observed between CR9501 and motavizumab in the context of the prefusion F trimer

characterized Fab-bound prefusion $\mathrm{F}$ trimers and one class composed of CR9501-bound trimers that appeared to be completely splayed open, tethered only by the trimerization motif (Fig. 5e). To verify that the closed conformation of prefusion $\mathrm{F}$ observed in the motavizumab-bound samples was not due to trimer-stabilizing effects of this antibody, we coexpressed monomeric prefusion $\mathrm{F}$ with motavizumab and analyzed the resulting complex by size-exclusion chromatography (Supplementary Fig. 6). This complex eluted at a volume corresponding to a Fab-bound monomer. In contrast, coexpression with the quaternary-specific antibody AM14 resulted in an elution profile corresponding to a Fab-bound trimer, consistent with published results ${ }^{40}$. Collectively, these results suggest that the trimeric and monomeric forms of soluble prefusion $\mathrm{F}$ exist in an equilibrium and demonstrate that binding of CR9501 favors trimer dissociation.

CR9501-bound $F$ resembles a natural prefusion state. To determine the structural basis for CR9501-enhanced trimer dissociation, we compared the CR9501-bound prefusion F monomer structure to a single protomer of a previously solved trimeric prefusion F structure (PDB ID: 4MMU). These structures were similar, with an RMSD of $1.7 \AA$ for $430 \mathrm{Ca}$ atoms. However, despite the high overall similarity, several differences were observed. A rigid-body motion between the trimer apex and the membrane-proximal region of the protein was present in the monomeric structure. This resulted in a change in the orientation of the $\alpha 1$ and $\alpha 5$ helices at the apex of the protein, and a small change in the orientation of antigenic site IV and the a10 helix at the base of the CR9501-bound monomer compared to the 4MMU structure. When three monomers from the CR9501-bound structure were used to model the prefusion $F$ trimer, a small interprotomeric clash between helices $\alpha 1$ and $\alpha 5$ of adjacent protomers was visible (Fig. 6a). Although it is possible that this clash could contribute to destabilization of the prefusion $\mathrm{F}$ trimer, it is not possible to determine if this clash is a result of CR9501 binding, or if this change in the apex of prefusion $\mathrm{F}$ simply represents a different conformation that can be adopted by monomeric prefusion $\mathrm{F}$ when it is not constrained by the presence of other protomers.

Because the crystal structures of multiple trimeric prefusion RSV F proteins have recently been solved, we compared these structures to determine if the conformational changes we observed are specific to the CR9501-bound structure. Combining the static snapshots from 26 previously solved prefusion $\mathrm{F}$ structures revealed trimer flexibility at both the trimer apex and the membrane-proximal region (Supplementary Movies 2 and 3). In each of the structures analyzed, the $\alpha 1$ and a5 helices adopt one of two distinct orientations (Fig. 6b, c, Supplementary Table 3). In the first orientation (State 1 ), the $\alpha 1$ and $\alpha 5$ helices are closely packed, with the membrane-distal region of the a5 helix from one protomer contacting both the $\alpha 1$ and a5 helices from the neighboring protomer. In this conformation, Ile217 is oriented toward the center of the trimer apex and shields access to the central cavity. In the second arrangement (State 2), the top portion of the $\alpha 5$ and $\alpha 1$ helices are tilted by approximately $20^{\circ}$ and $10^{\circ}$, respectively, resulting in a decrease in the interprotomeric contacts between $\alpha 1$ 
a

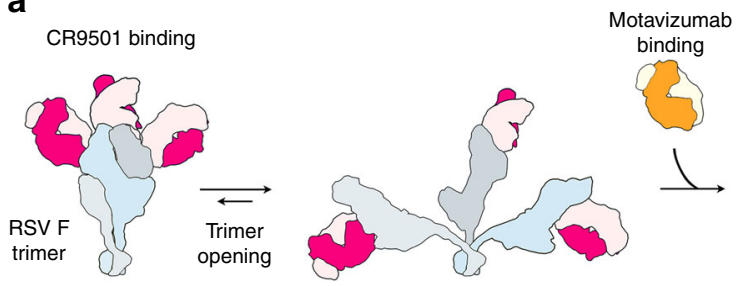

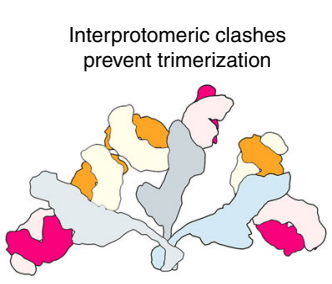

C

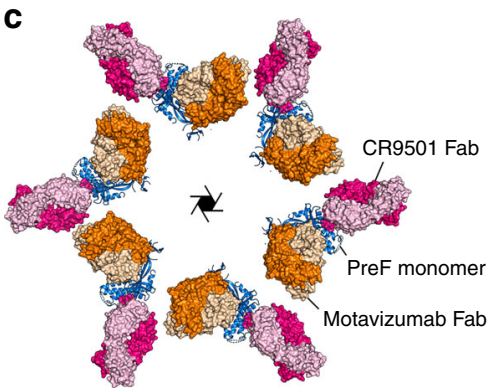

Molecular replacement solution found in $P 6$
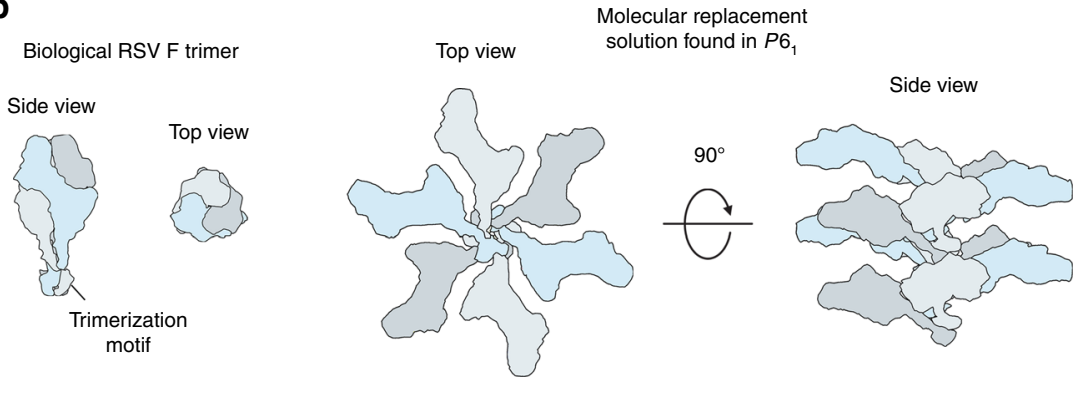

d

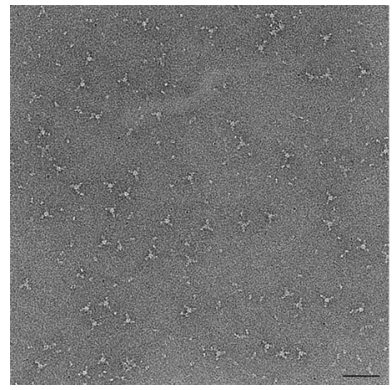

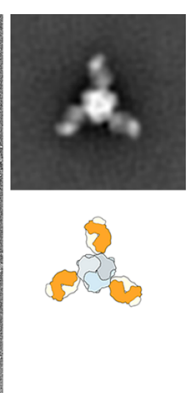

e

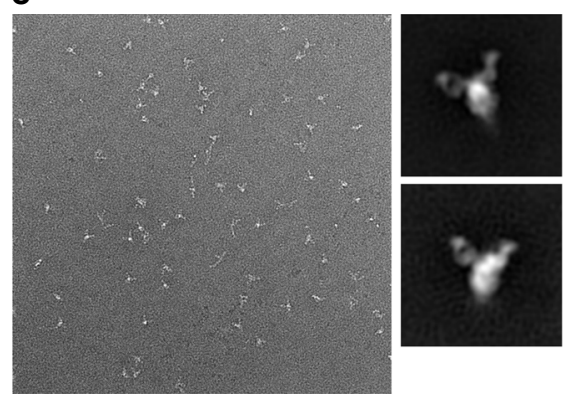

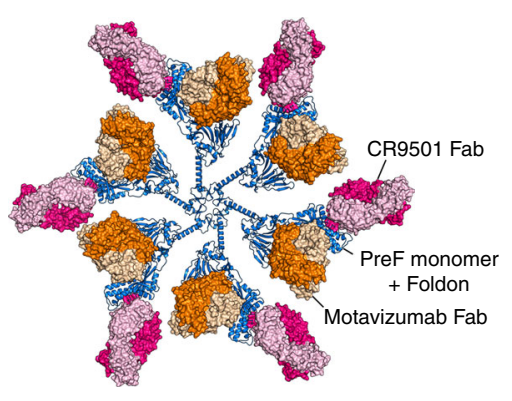
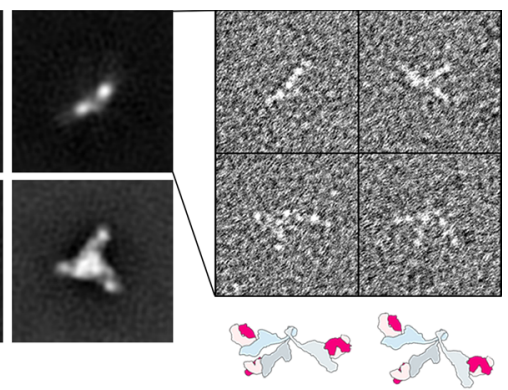

Fig. 5 Structural evidence for CR9501-enhanced opening of prefusion F trimers. a Model illustrating the strategy used to generate trimeric prefusion $\mathrm{F}$ bound simultaneously by CR9501 and motavizumab, showing prefusion F protomers in blue, light gray and dark gray, CR9501 in pink and light pink, and motavizumab in orange and tan. $\mathbf{b}$ Cartoon depicts the biological RSV F trimer (left) and the molecular replacement solution obtained in space group P6 1 (right), shown without Fabs for clarity. Prefusion F protomers are colored blue, light gray, and dark gray. c The molecular replacement solution from the crystal structure of prefusion F bound to CR9501 and motavizumab, with six copies of the asymmetric unit shown and the sixfold screw axis indicated in the center (top). Prefusion F is shown as blue ribbons and motavizumab and CR9501 Fabs are shown as molecular surfaces. CR9501 heavy and light chains are colored dark pink and light pink and the motavizumab Fab heavy and light chains are colored orange and tan, respectively. The Foldon motif was modeled on to the C-terminus of each monomer, using a previously determined prefusion F structure in which this domain is visible (PDB ID: 4MMV) (bottom). d Negative-stain EM of the prefusion F-motavizumab complex showed primarily top-down views of the closed, trimeric complex (scale bar = $50 \mathrm{~nm}$ ). A representative class average shows a closed trimer with three Fabs bound and the cartoon illustrates the orientation viewed in the class average. e Negative-stain EM of the prefusion F-CR9501 complex revealed multiple particle classes. Three representative class averages show different orientations of the closed, trimeric complex, whereas the fourth comprised individual monomers within splayed-open trimers. The inset shows a subset of the splayedopen trimers used to generate the fourth class average (right) and the cartoons illustrate the orientation of the splayed-open trimer thought to be present in the particles shown

and $\alpha 5$. In this state, Ile217 is oriented away from the central threefold axis and no longer shields the central cavity. Analysis of representative trimers from each state using the PDBePISA server showed that a State 1 trimer buries an average of $2331 \AA^{2}$ in each interprotomeric interface, with a predicted $\Delta G=-18.5 \mathrm{kcal} \mathrm{mol}^{-1}$, whereas a State 2 trimer buries $1928 \AA^{2}$, with a predicted $\Delta G=$ $-15.0 \mathrm{kcal} \mathrm{mol}^{-1}$. The membrane-proximal region of the prefusion F trimer also displayed a small degree of flexibility, with a continuum of orientations in which the a 10 helix shifts by up to $2 \AA$ away from the central threefold axis of the trimer (Fig. 6c). The crystal structures in which prefusion $\mathrm{F}$ adopted the State 2 conformation all display some degree of movement of the a 10 helix away from the central threefold axis, suggesting that changes at the trimer apex may be coupled to those in the membraneproximal region. In addition, the position of the CR9501 CDR H3 between two portions of the $\mathrm{F}_{2}$ subunit results in a small movement of this loop away from the $\alpha 1$ helix compared with the previously determined structure. Comparison to two other RSV F structures (PDB IDs: 4JHW and 4MMT) indicated that although the $\mathrm{F}_{2}$ subunit exists in multiple conformations, the degree of movement away from the $\alpha 1$ helix is largest in CR9501-bound RSV F (Fig. 6c).

Comparing the rigid-body movements identified in the CR9501-bound monomer to those of the two prefusion $\mathrm{F}$ states identified above revealed that the conformation of the CR9501bound monomer most closely resembles that of the State 2 trimer (Fig. $6 \mathrm{a}-\mathrm{c}$ ). The $\alpha 1$ and $\alpha 5$ helices are tilted away from the apex of the neighboring protomer, although to a lesser degree than what was observed for trimeric proteins, resulting in loss of interprotomeric contacts between $\alpha 1$ and $\alpha 5$ and orientation of Ile217 outward to expose the central cavity. Consistent with the subtle 
a

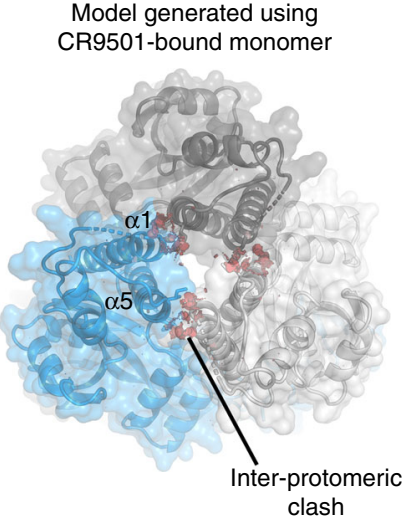

b

Prefusion F State 1

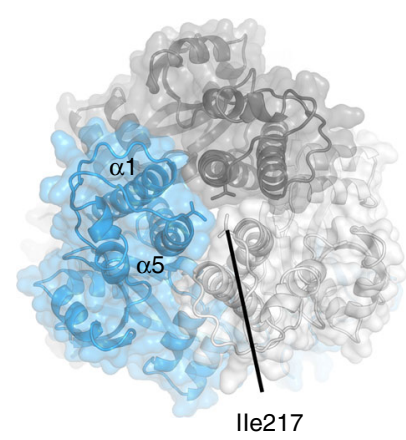

Prefusion F State 2

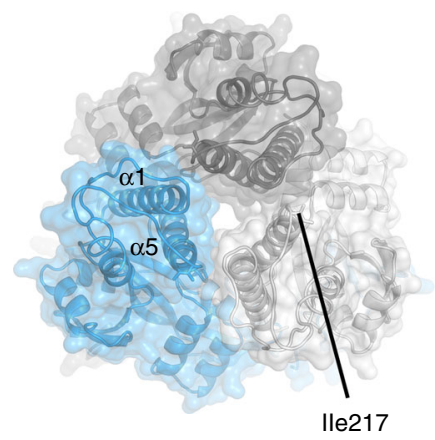

C
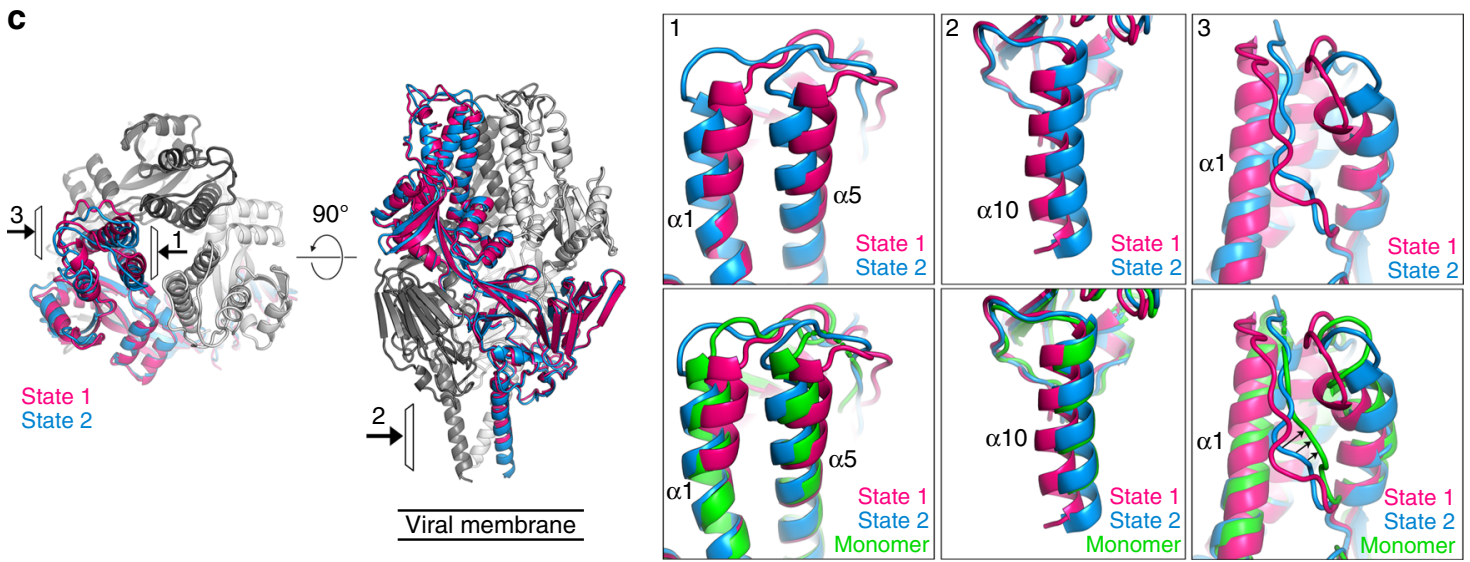

Fig. 6 CR9501-bound F resembles a naturally occurring prefusion state. a C3 symmetry was applied to the monomeric prefusion F-CR9501 structure to generate a prefusion $\mathrm{F}$ trimer. The resulting trimer is shown as ribbons and transparent molecular surfaces, with the three protomers colored blue, gray, and white, viewed looking toward the viral membrane. Predicted clashes between protomers are indicated with red. $\mathbf{b}$ Two states of prefusion $\mathrm{F}$ identified in 26 previously determined structures are shown and colored as in (a). c The two states of prefusion $\mathrm{F}$ are shown in pink and blue ribbons and viewed looking toward (left) or along (right) the viral membrane. The top insets show the views denoted by the arrows at (1) the trimer apex viewed from the central cavity, (2) the heptad repeat B and (3) the $F_{2}$ subunit. The bottom insets are the same but include the monomer from the CR9501-bound structure shown in green

differences between the CR9501-bound monomer and the trimeric prefusion F structures, CR9501 displayed high-affinity binding for both trimeric and monomeric prefusion F (Supplementary Fig. 7). Together these analyses suggest that the rigidbody motion observed in the CR9501-bound monomer represents a naturally occurring conformation of prefusion $\mathrm{F}$ rather than a CR9501-induced change, and that there are at least two conformations of the prefusion $\mathrm{F}$ trimer, one of which has reduced interprotomeric stability.

RSV F trimers open and dissociate at the cell surface. The unique competition profiles observed for CR9501 and motavizumab on trimeric and monomeric variants of prefusion $\mathrm{F}$ suggested that CR9501 could be used as a reagent to probe trimer opening of fulllength RSV F on the surface of cells. FreeStyle 293-F cells were transfected with a vector encoding full-length wild-type $F$ and incubated with competitor Fab or buffer before being incubated with an CF-647-labeled IgG. CR9501 Fab preincubation reduced the signal observed in cells stained with CF-647-labeled CR9501 IgG by nearly $80 \%$ compared with buffer-only preincubation (Fig. 7a, dark pink). A similar result was observed for motavizumab Fab preincubation before staining with CF-647-labeled motavizumab IgG (Fig. 7a, orange). Preincubation of the cells with CR9501 Fab blocked only $\sim 30 \%$ of CF-647-labeled motavizumab IgG binding (Fig. 7a, yellow), consistent with the model above in which
CR9501 binding favors opening of the trimer and relieves the interprotomeric clash with motavizumab. However, in contrast to what was observed with soluble trimeric prefusion $\mathrm{F}$ proteins, motavizumab preincubation blocked binding of CF-647-labeled CR9501 IgG by less than 20\% (Fig. 7a, light pink). These results indicate that the full-length $\mathrm{F}$ protein on the surface of cells samples an open conformation compatible with the binding of both CR9501 and motavizumab.

To verify that the stabilizing mutations present in the soluble proteins were not responsible for order-of-addition dependence observed previously, these same stabilizing mutations were incorporated into full-length $\mathrm{F}$ and the resulting variants were expressed on the surface of cells. Similar to the wild-type protein, very little competition was observed between CR9501 and motavizumab for either order of antibody addition (Fig. 7a). To determine if this phenomenon was restricted to lab-adapted RSV strains, we tested two additional variants of the full-length proteins that were generated using the $\mathrm{F}$ protein sequences from two clinical isolates of RSV. CR9501 and motavizumab again showed low levels of competition for binding to cells expressing these variants (Fig. 7a).

Our results thus far indicated that full-length RSV F samples a conformation or oligomeric state on the surface of cells compatible with the binding of both antibodies. However, our data could not yet distinguish between a model in which RSV F is partially open or completely dissociated (monomeric), which 
a

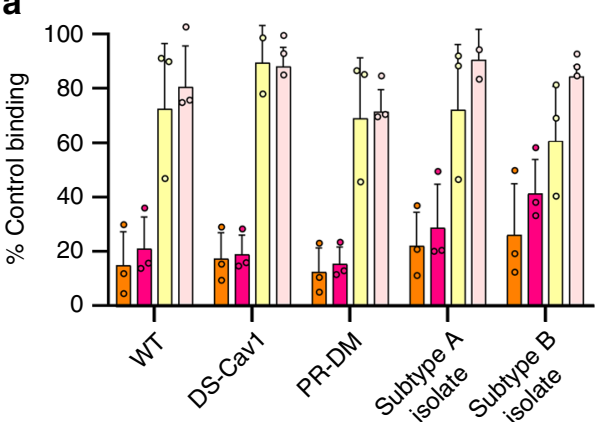

b

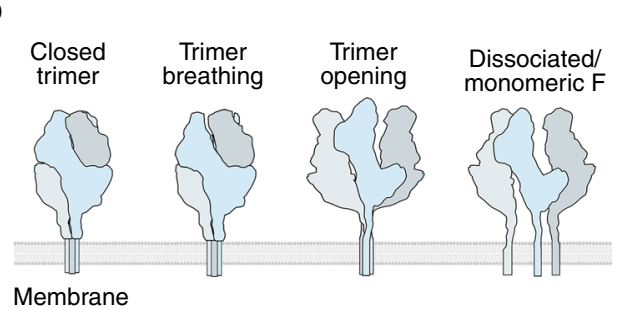

C
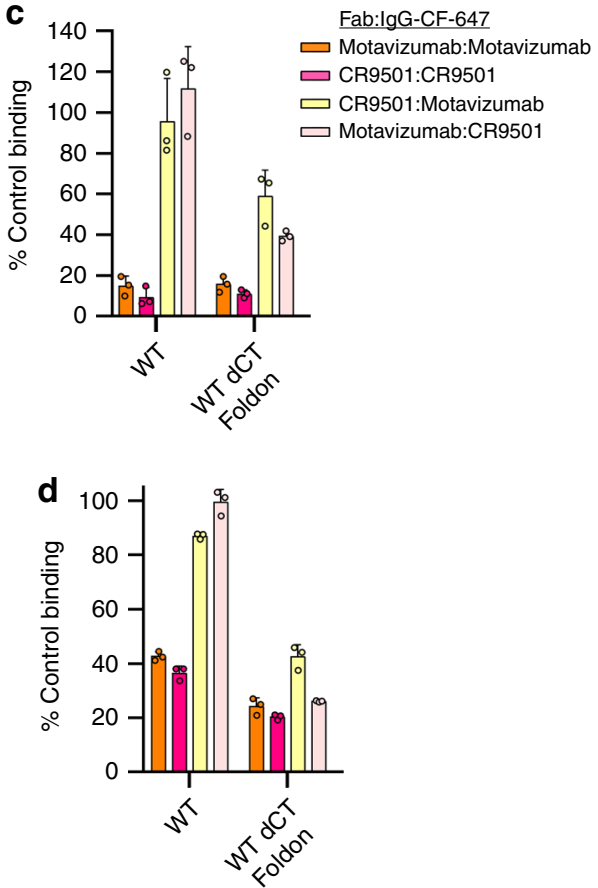

Fig. 7 RSV F trimers open and dissociate at the cell surface. a Full-length RSV F proteins were expressed on the surface of FreeStyle 293-F cells and incubated with either CR9501 Fab or motavizumab Fab. Cells were then stained with CF-647-labeled CR9501 IgG or CF-647-labeled motavizumab lgG and binding was analyzed by flow cytometry. b A cartoon depicts prefusion F protomers in blue, light gray, and gray and shows four possible states of the prefusion $\mathrm{F}$ trimer, two of which could allow simultaneous binding of motavizumab and CR9501. c Full-length RSV F or a variant in which the cytoplasmic tail was replaced with a Foldon trimerization motif was expressed on the surface of FreeStyle 293-F cells and stained as in (a). d Same as (c), but with RSV $\mathrm{F}$ and the Foldon variant expressed on the surface of HeLa cells. Bar graphs show the mean and error bars indicate the standard deviation ( $n=3$ biologically independent experiments with two technical replicates)

largely depends upon whether the transmembrane domains are associated (Fig. 7b). Thus, we performed an additional experiment in which the cytoplasmic tail of $\mathrm{F}$ was replaced with a Foldon trimerization motif, and CR9501 and motavizumab competition was again measured on the surface of HEK293-F cells (Fig. 7c). We reasoned that if prefusion $\mathrm{F}$ trimers are partially open on the surface of cells, inclusion of this domain should not impact the competition of CR9501 and motavizumab, since it would be redundant with the presence of the associated transmembrane domains. In contrast, if the trimers dissociated at the cell surface, inclusion of the Foldon domain should increase competition between CR9501 and motavizumab, since Foldon would favor trimerization of the protomers. When $\mathrm{F}$ was fused to the Foldon motif, increased competition between CR9501 and motavizumab was observed (Fig. 7c, yellow and pink), indicating that a fraction of prefusion $\mathrm{F}$ is monomeric at the cell surface.

Although competition between CR9501 and motavizumab was higher in the Foldon-fused variant than in wild-type RSV F, binding was not reduced to the level observed for the control samples (Fig. 7c, orange and dark pink), suggesting that partial trimer opening also occurs. To verify that this property was not restricted to suspension cell lines, we repeated the competition assay with RSV F and the Foldon-fused variant expressed on the surface of adherent HeLa cells and obtained similar results (Fig. 7d). To rule out the possibility that RSV F is entirely monomeric when expressed at the cell surface, we measured binding to RSV F-transfected cells of the quaternary-specific antibody $\mathrm{AM} 14^{40}$ and a prefusion-specific antibody $\left(\mathrm{AM} 22^{9}\right)$ that can bind to both monomeric and trimeric F (Supplementary Fig. 8). We reasoned that if there were a static pool of monomeric RSV F at the cell surface, the AM14 signal would be much lower than that of AM22. However, AM14 binding was similar to
AM22, indicating that full-length $\mathrm{F}$ is in a monomer-trimer equilibrium at the cell surface.

\section{Discussion}

The biochemical assays and structural analysis presented here demonstrate that CR9501 represents a new class of RSVneutralizing antibody that enhances dissociation of trimeric prefusion $\mathrm{F}$ while also preventing the conformational changes required for conversion of prefusion $\mathrm{F}$ into the postfusion conformation. We have recently determined that a large proportion of the neutralizing antibody response in healthy adults target this prefusion-specific antigenic site ${ }^{23}$, but only one other site $\mathrm{V}$ reactive antibody-hRSV90-has been structurally characterized thus far ${ }^{26}$. Although both CR9501 and hRSV90 contact antigenic site V, CR9501 is rotated by $\sim 60^{\circ}$ compared with hRSV90 (Supplementary Fig. 9), resulting in substantial contacts with the $\mathrm{F}_{2}$ subunit. Similar effects on trimerization were not reported for hRSV90, indicating that the additional contacts made between CR9501 and the $F_{2}$ subunit could be responsible for the trimer disassembly observed here. Previous studies have implicated the $\mathrm{F}_{2}$ subunit in species specificity of RSV and in fusogenicity of $F^{31,32}$. However, the conformational changes observed in the CR9501-bound monomer are subtle, in contrast to what has been observed in monomeric structures of antibody-bound influenza hemagglutinin (HA) in which conformational changes indicative of triggering are present ${ }^{41-44}$. This is likely because CR9501 also contacts the $\alpha 2-\alpha 3$ helices, which would prevent conformational changes associated with fusion. It therefore remains possible that interactions with the $\mathrm{F}_{2}$ subunit are linked to monomerization, triggering, or both, but these changes could be countered in the CR9501-bound structure by the stabilizing contacts made with other regions of the protein. 
Our analysis of the many previously determined RSV F structures suggests that the trimeric, prefusion $\mathrm{F}$ protein samples at least two conformations, one of which is less compact and could favor trimer opening. The small changes that occur between the two states of the prefusion $\mathrm{F}$ trimer are reminiscent of the breathing motions that have been described for the envelope (Env) protein of HIV-1, which have been observed in soluble proteins and on the surface of virions ${ }^{45,46}$. This conformational flexibility is required for binding of the HIV-1 receptor and coreceptor, which prime Env for fusion ${ }^{45-47}$. Mutations that stabilize the trimeric interface at the base of Env can limit breathing at the trimer apex and prevent the conformational changes required for receptor binding ${ }^{48}$. Antibodies that cause Env trimer dissociation have been described and it was proposed that binding of these antibodies may depend on the same breathing motions required for receptor binding ${ }^{49}$. Similar to CR9501, these antibodies neutralize HIV-1 and recognize both monomeric and trimeric Env proteins, suggesting that susceptibility to antibody-induced changes in oligomeric state may be a common feature among class I fusion proteins, particularly those that exhibit conformational flexibility.

Prefusion ectodomains of RSV F, as well as a number of other class I fusion proteins, are monomeric in the absence of a trimerization domain, suggesting that interprotomeric interactions are weak in these proteins $s^{9,37,38}$. It has therefore been assumed that the restriction of the protomers within the $2 \mathrm{D}$ membrane increases the local concentration of these proteins sufficiently to favor trimerization. Our results suggest that for RSV F this may not always be true, and that prefusion $F$ exists in a monomer-trimer equilibrium, even when embedded in a lipid bilayer. We note that cryo-electron tomography studies of budding RSV particles have only identified density consistent with trimeric prefusion $\mathrm{F}$ at the surface of filamentous viral particles $^{50,51}$. This may be because monomeric proteins are present at the viral surface but are too small to be visualized by tomography, or that the RSV matrix protein layer, present $\sim 5 \mathrm{~nm}$ below the viral membrane, plays a role in the trimerization of RSV F. This would be consistent with previous studies suggesting that the matrix protein and the $\mathrm{F}$ cytoplasmic tail interact ${ }^{52}$. Interestingly, a monomer-specific antibody isolated against HA binds to influenza-infected cells $s^{53}$, indicating that HA may also sample an open conformation at the cell membrane. This finding suggests that trimer opening at the cell surface may not be unique to RSV and could represent a previously unappreciated property of certain class I fusion proteins. These results also have implications for RSV vaccine design because several leading candidates are based on trimeric prefusion-stabilized $\mathrm{F}$ antigens. Studies will be needed to assess the relationship between transient trimer opening and immunogenicity.

\footnotetext{
Methods

Identification of anti-RSV F antibodies. Neutralizing monoclonal antibodies against RSV F protein were recovered from mature B cells isolated from PBMCs obtained through the San Diego Blood Bank. Samples obtained from human volunteers followed protocols approved by the San Diego Blood Bank Review Board and informed consent was obtained from the donors prior to the blood donation. In short, $\mathrm{CD} 22^{+} \mathrm{B}$ cells were isolated by immunomagnetic positive selection (Miltenyi) from donor PBMCs and transformed with Epstein-Barr virus, followed by stimulation for 5 days with $\mathrm{CpG}$ and recombinant human interleukin 2 (IL-2). Two weeks later, supernatants were tested by enzyme-linked immunosorbent assay (ELISA) against RSV F-transfected cells. Variable-heavy (VH) and variable-light (VL) chain genes were cloned from oligoclonal B-cell cultures corresponding to antigen-reactive wells using pooled primers described in patent application US20130177573A1, and specificity of the cloned antibodies was confirmed by ELISA.
}

Expression of antibodies utilized in neutralization assays. The sequences obtained from each VH and VL of the antibody clones were codon-optimized for expression in human cells at GeneArt/Invitrogen. The variable regions of these functional variants were subsequently cloned directly by restriction digest for expression in the IgG expression vectors pCP9-kappa and pCP9-gamma. Vectors encoding the IgGs were transfected into PER.C6 ${ }^{\circledR}$ cells and the antibodies were purified from cell supernatants using POROS Mabcapture A chromatography (Applied Biosystems). The antibodies were then exchanged into $50 \mathrm{mM}$ sodium acetate, $50 \mathrm{mM}$ sodium chloride, $\mathrm{pH} 5.5$. The quality of each antibody was confirmed by size-exclusion chromatography, SDS-PAGE and isoelectric focusing.

Neutralization assay. RSV-susceptible VERO cells (ATCC-CCL-81) were seeded at a density of $3 \times 10^{4}$ cells well $^{-1}$ in a 96-well format. The following day 1200 plaque-forming units of virus were incubated with serial dilutions of the RSV antibodies for $1 \mathrm{~h}$ at $37^{\circ} \mathrm{C}$ before addition to the cells and incubation for $24 \mathrm{~h}$. The media was then refreshed, and the cells were incubated for $48 \mathrm{~h}$. The cells were fixed with $80 \%$ acetone and virus growth was detected using a biotinylated anti-F protein antibody coupled with HRP-conjugated streptavidin. The wells were washed again, and turnover of the 3,3',5,5'-tetramethylbenzidine substrate was measured at an optical density of $450 \mathrm{~nm}\left(\mathrm{OD}_{450}\right)$. VNA titers were calculated as the antibody concentration that caused $50 \%$ reduction in the $\mathrm{OD}_{450}$, expressed as $\mathrm{IC}_{50}$. Four biologically independent experiments with nine technical replicates were performed for CR9501 and palivizumab and one independent experiment with 27-43 technical replicates was performed for D25.

RSV F mutation library and immunofluorescence assay. Epitope mapping by mutagenesis was performed by Integral Molecular. A shotgun mutagenesis library of RSV F was created using a previously published method ${ }^{54}$. The parental plasmid expressing RSV F A2 strain (GenBank: FJ614814.1) polyprotein was used as a template to make a library of random mutations across RSV F, created using PCRbased mutagenesis (Diversify PCR Random Mutagenesis Kit, Clontech) plus 39 additional mutants. Each mutant clone was sequence verified. A complete mutation library was assembled by selection of at least two mutant clones per residue, preferably representing a conserved and nonconserved residue at each position.

Mutation libraries and controls were expressed in human HEK-293T cells (ATCC-CRL-3216). Twenty-two hours posttransfection cells were washed and fixed in $4 \%$ (wt/vol) paraformaldehyde, incubated with $0.5 \mu \mathrm{gmL}^{-1}$ of CR9501 or CR9502 $\mathrm{IgG}^{33}$, followed by AlexaFluor 488-conjugated secondary antibody (Jackson ImmunoResearch). Microplates were measured using the Intellicyt HTFC screening system. Antibody reactivities against each mutant F protein clone were calculated relative to wild-type protein reactivity by subtracting the signal from mock-transfected controls and normalizing to the signal from wild-type Ftransfected controls. Binding of CR9501 was also compared with a polyclonal serum, palivizumab, and CR9502, representing different epitopes, to confirm that the expressed mutant F proteins were not misfolded.

\section{Antibody production for crystallization, SPR, and cytometry. Plasmids} encoding the heavy and light chain of CR9501 or motavizumab antibodies were cotransfected into FreeStyle-293F cells (ThermoFisher Scientific, Cat. No. R79007). Cell supernatants were harvested after 6 days and antibodies were purified using protein A resin, followed by size-exclusion chromatography using a Superdex 200 column (GE Healthcare) in buffer containing $2 \mathrm{mM}$ Tris $\mathrm{pH} 8.0,200 \mathrm{mM} \mathrm{NaCl}$ and $0.02 \% \mathrm{NaN}_{3}$ or phosphate-buffered saline (PBS) with $0.02 \% \mathrm{NaN}_{3}$.

To generate motavizumab and CR9501 Fabs, a $1 \mathrm{mg} \mathrm{mL}^{-1}$ solution of each antibody was digested with 1:4000 (wt/wt) of Lys-C overnight at $37^{\circ} \mathrm{C}$. Undigested antibody and digested Fc were separated from Fabs using protein A resin, and the flow-through was further purified by size-exclusion chromatography using a Superdex 75 column (GE Healthcare) in buffer containing $2 \mathrm{mM}$ Tris $\mathrm{pH} 8.0$, $200 \mathrm{mM} \mathrm{NaCl}$ and $0.02 \% \mathrm{NaN}_{3}$ or phosphate-buffered saline with $0.02 \% \mathrm{NaN}_{3}$.

Production of protein complexes for crystallization and SPR. Trimeric prefusion F (PR-DM) ${ }^{33}$ comprised residues 1-513 from RSV F strain A2 with N67I/S215P mutations and a C-terminal fibritin trimerization motif (Foldon), HRV 3C cleavage site, $8 \times$ His-tag and StrepTagII. A second variant of trimeric prefusion F called DS Cav $1^{39}$ is composed of residues 1-513 from RSV F strain A2 with S155C/S290C/ S190F/V207L substitutions and a C-terminal Foldon trimerization motif, thrombin cleavage site, $8 \times$ His-tag and StrepTagII. Monomeric prefusion F is identical to DS Cav1 but lacks the Foldon trimerization motif. Vectors encoding each were trans fected into FreeStyle 293-F cells (ThermoFisher Scientific, Cat. No. R79007). For proteins utilized in crystallization, kifunensine was added to the cell suspension approximately $4 \mathrm{~h}$ after transfection to a final concentration of $5 \mu \mathrm{M}$. Proteins were purified from cell supernatants using StrepTactin resin (IBA), then digested with $5-10 \%$ (wt/wt) EndoH to remove $N$-linked glycans, followed by cleavage with 10 units $\mathrm{mg}^{-1}$ of HRV $3 \mathrm{C}$ to remove tags. The protein was further purified by size-exclusion chromatography using a Superdex 200 column (GE Healthcare) in buffer containing $2 \mathrm{mM}$ Tris pH 8.0, $200 \mathrm{mM} \mathrm{NaCl}$ and $0.02 \% \mathrm{NaN}_{3}$.

Ternary prefusion F-motavizumab-CR9501 complex was generated by first incubating PR-DM with a 1.5-fold molar excess of CR9501 Fab at room temperature for $1 \mathrm{~h}$, followed by the addition of 1.5-fold molar excess of motavizumab Fab and incubation at room temperature for $30 \mathrm{~min}$. The complexes were then separated from excess Fabs using a Superdex 200 Increase column (GE Healthcare). The monomeric 
prefusion F-CR9501 complex was produced by incubating monomeric DS-Cav1 with a 1.5-fold molar excess of CR9501 Fab before separating the complex from excess Fabs using a Superdex 200 Increase column (GE Healthcare).

Analytical size-exclusion chromatography. PR-DM or DS-Cav1 were incubated with a 1.5-fold molar excess of CR9501 Fab at room temperature for $1 \mathrm{~h}$, followed by the addition of 1.5 -fold molar excess of motavizumab Fab and incubation at room temperature for $30 \mathrm{~min}$. Complexes were also generated by first incubating PR-DM or DS-Cav1 with motavizumab Fab for $1 \mathrm{~h}$ before incubation with CR9501 for $30 \mathrm{~min}$. For simultaneous addition, both Fabs were incubated with PR-DM or DS-Cav1 for $30 \mathrm{~min}$. The complexes were then analyzed using a Superdex 200 Increase column (GE Healthcare) in buffer containing $2 \mathrm{mM}$ Tris pH 8.0, $200 \mathrm{mM}$ $\mathrm{NaCl}$ and $0.02 \% \mathrm{NaN}_{3}$.

Monomeric prefusion F (DS-Cav1) was coexpressed with either motavizumab Fab or AM14 Fab and furin in FreeStyle 293-F cells (ThermoFisher Scientific, Cat. No. R79007) and purified from cell supernatants using Ni-NTA resin (ThermoScientific). The complexes were then analyzed using a Superdex 200 Increase column (GE Healthcare) in buffer containing $2 \mathrm{mM}$ Tris $\mathrm{pH} 8.0,200 \mathrm{mM} \mathrm{NaCl}$ and $0.02 \% \mathrm{NaN}_{3}$.

Crystallization and data collection. The monomeric prefusion F-CR9501 complex was crystallized by sitting-drop vapor diffusion by mixing $100 \mathrm{~nL}$ of protein $\left(4.2 \mathrm{mg} \mathrm{mL}^{-1}\right)$ with $50 \mathrm{~nL}$ of a reservoir solution composed of $14.3 \%(\mathrm{w} / \mathrm{v})$ polyethylene glycol (PEG) 8000, 2.4\% (v/v) 2-methyl-2,4-pentanediol (MPD), $0.1 \mathrm{M}$ sodium citrate $\mathrm{pH} 5.5$ and $0.1 \mathrm{M}$ sodium malonate $\mathrm{pH}$ 7.0. Crystals were soaked in reservoir solution supplemented with $30 \%(\mathrm{v} / \mathrm{v})$ ethylene glycol and plunge frozen in liquid nitrogen. Data were collected to $3.3 \AA$ at the SBC beamline 19-ID using a wavelength of $0.9792 \AA$ (Advanced Photon Source, Argonne National Laboratory).

Crystals of the ternary prefusion F-motavizumab-CR9501 complex were produced by hanging-drop vapor diffusion by mixing $0.67 \mu \mathrm{L}$ of protein $(4.69 \mathrm{mg}$ $\mathrm{mL}^{-1}$ ) with $1.33 \mu \mathrm{L}$ of a reservoir solution containing $30 \%(\mathrm{v} / \mathrm{v})$ PEG $400,0.19 \mathrm{M}$ ammonium sulfate, $3.1 \%(\mathrm{w} / \mathrm{v})$ PEG 8000 and $0.1 \mathrm{M}$ Tris $\mathrm{pH}$ 8.5. Crystals were transferred into a solution containing reservoir supplemented with $20 \%(\mathrm{v} / \mathrm{v})$ glycerol and suspended over an identical reservoir solution for approximately $9 \mathrm{~h}$ before plunge freezing in liquid nitrogen. Diffraction data were collected to $4.1 \AA$ at the SBC beamline 19-ID using a wavelength of $0.9793 \AA$ (Advanced Photon Source, Argonne National Laboratory).

Structure determination, model building, and refinement. Software used for processing of X-ray diffraction data was curated by SBGrid and accessed through the CCP4i interface ${ }^{55-57}$. Data were indexed and integrated using iMOSFLM ${ }^{58}$ before merging and scaling with AIMLESS ${ }^{59}$. PHASER ${ }^{60}$ was used to identify molecular replacement solutions, and the structures were refined using PHENIX ${ }^{61}$ and built manually using $\operatorname{Coot}^{62}$. Data collection and refinement statistics for the two crystal structures are shown in Supplementary Table 2 and a stereo image of a portion of the electron density map is shown in Supplementary Fig. 10.

The monomeric prefusion F-CR9501 complex crystallized in space group $P 2_{1} 2_{1} 2_{1}$ and a molecular replacement solution was found using a prefusion $\mathrm{F}$ protomer (PDB ID: 5EA4), and the CR9501 Fab from the ternary complex as search models. The asymmetric unit contained two prefusion $\mathrm{F}$ protomers and two CR9501 Fabs. The model was built manually in Coot and refined in PHENIX using NCS torsion restraints to an $R_{\text {work }} / R_{\text {free }}$ of $21.2 / 25.9 \%$.

The ternary prefusion F-motavizumab-CR9501 complex formed crystals in space group $P 6_{1}$, and a molecular replacement solution was found using one prefusion F protomer (PDB ID: 5C69), one motavizumab Fab (PDB ID: 3QWO), the light chain from D25 (PDB ID: 4JHA), and the heavy chain from N60-I3 Fab (PDB ID: 4RFO) as search models. The asymmetric unit contained one prefusion $F$ protomer, one motavizumab Fab, and one CR9501 Fab. Electron density was not present for the membrane-proximal region of prefusion $\mathrm{F}$ and therefore this portion of the structure is absent in our model. The remainder of the model was built manually in Coot and refined in PHENIX using reference-model restraints to an $R_{\text {work }} / R_{\text {free }}$ of $26.2 / 30.6 \%$.

Negative-stain electron microscopy. Carbon-coated grids (EMS) were glowdischarged before $4.8 \mu \mathrm{L}$ of PR-DM-CR9501 complex or PR-DM-motavizumab complex $\left(0.02 \mathrm{mg} \mathrm{mL}^{-1}\right)$ was applied to the grid and allowed to incubate for $30 \mathrm{~s}$. The grids were rinsed with a buffer containing $2 \mathrm{mM}$ Tris $\mathrm{pH} 8.0,200 \mathrm{mM} \mathrm{NaCl}$, $0.02 \% \mathrm{NaN}_{3}$ and stained with methylamine tungstate (Nano-W $\mathrm{W}^{\circledR}$, Nanoprobes). Images were acquired using an FEI Talos operated at $200 \mathrm{kV}$, equipped with an FEI Ceta $16 \mathrm{M}$ camera $(4 \mathrm{k} \times 4 \mathrm{k})$. Image processing and $2 \mathrm{D}$ classifications were carried out using cisTEM ${ }^{63}$. Initial classification was performed to remove junk particles, followed by averaging into 15 (PRDM-CR9501), 10 (PRDM-motavizumab), or 10 (PRDM-CR9501-motavizumab) classes.

Surface plasmon resonance competition assay. Biacore X100 (GE Healthcare) was used to measure the binding of CR9501 Fab and motavizumab Fab to trimeric prefusion F (both DS-Cavl and PR-DM variants) or monomeric prefusion F (DSCavl variant). Proteins were captured using an NTA sensor chip, which was regenerated between each cycle using $0.35 \mathrm{M}$ ethylenediaminetetraacetic acid (EDTA) followed by $0.5 \mathrm{mM} \mathrm{NiCl}_{2}$. For each variant, five different sets of injections were performed using $200 \mathrm{nM}$ solution for all Fabs and HBS-P+ pH 8.0 for buffer: (1) buffer, buffer, buffer; (2) buffer, buffer, CR9501; (3) buffer, buffer, motavizumab; (4) buffer, motavizumab, CR9501; (5) buffer, CR9501, motavizumab. The data were double-reference subtracted using the buffer-only reference samples.

Cell-surface expression of $\mathbf{F}$ and flow cytometric analysis. Vectors encoding full-length wild-type RSV F variants, variants containing the DS-Cav1 or PR-DM stabilizing mutations, or a variant in which the cytoplasmic tail was replaced with a Foldon trimerization motif were cotransfected with a plasmid encoding furin in FreeStyle 293-F cells (ThermoFisher Scientific, Cat. No. R79007) or HeLa cells (ATCC-CCL-2). After approximately $24 \mathrm{~h}$, cells were washed with blocking buffer (PBS with $0.5 \%$ bovine serum albumin (BSA), $1 \mathrm{mM}$ EDTA), and incubated with a $500 \mathrm{nM}$ solution of CR9501 Fab or motavizumab Fab for $30 \mathrm{~min}$. For HeLa cells, adherent cells were stained on the surface of 48 -well plates using PBS with $0.5 \%$ BSA Solutions containing $1 \mathrm{mg} \mathrm{mL}^{-1}$ of motavizumab IgG or CR9501 IgG in $2 \mathrm{mM}$ Tris $\mathrm{pH} 8.0,200 \mathrm{mM} \mathrm{NaCl}$ and $0.02 \% \mathrm{NaN}_{3}$ were fluorescently labeled using the Mix-nStain ${ }^{\mathrm{TM}} \mathrm{CF}^{\circledast} 647$ antibody labeling kit (Sigma) according to the manufacturer's instructions and a $100 \mathrm{nM}$ solution of labeled IgGs was added to the cells and incubated for $20 \mathrm{~min}$. HeLa cells were then removed from 48-well plates using PBS with $0.5 \% \mathrm{BSA}$ and $5 \mathrm{mM}$ EDTA. Cells were resuspended in a solution containing a 1:2000 dilution of SYTOX Blue Dead Cell Stain (Molecular Probes) before analysis using a MACSQuant (Miltenyi) or a LSRFortessa SORP Flow Cytometer (BD). Due to lower levels of $\mathrm{F}$ protein expression in HeLa cells, these cells were gated on positive CF-647 signal before analysis. Experiments shown are the result of duplicate measurements for three biologically independent experiments.

For monoclonal antibody binding, a $500 \mathrm{nM}$ solution of motavizumab, CR9501, AM22, AM14 or ADI-15576 IgG in blocking buffer was added to cells and incubated for $30 \mathrm{~min}$. Cells were washed and a 1:300 dilution of mouse anti-human Fc-PE (SouthernBiotech, Cat. No. 9040-09) was added and incubated for $20 \mathrm{~min}$ Cells were resuspended in a solution containing a 1:2000 dilution of SYTOX Blue Dead Cell Stain (Molecular Probes) before analysis using a LSRFortessa SORP Flow Cytometer (BD).

Surface plasmon resonance affinity measurements. Trimeric or monomeric DS-Cavl, each with a C-terminal His tag, were immobilized on an NTA sensor chip to approximately 175 or 150 response units (RU), respectively, per cycle using a Biacore X100 (GE Healthcare). The sensor chip was regenerated between cycles using $0.25 \mathrm{M}$ EDTA followed by $0.5 \mathrm{mM} \mathrm{NiCl}_{2}$. A buffer-only sample was injected over the sample and reference flow cells, followed by CR9501 Fab threefold serially diluted from 100 to $0.4 \mathrm{nM}$ in HBS-P+, with a duplication of the $3.7 \mathrm{nM}$ concentration. The data were double-reference subtracted and fit to a 1:1 binding model using Scrubber2.

Reporting summary. Further information on research design is available in the Nature Research Reporting Summary linked to this article.

\section{Data availability}

Atomic coordinates and structure factors for the monomeric prefusion RSV F-CR9501 Fab structure and the ternary prefusion RSV F-CR9501 Fab-motavizumab Fab structure have been deposited with the Protein Data Bank under accession codes 6OE4 and $6 \mathrm{OE} 5$, respectively. The authors declare that all other data supporting the findings of this study are available within the article and its Supplementary Information files, or are available from the authors upon request.

\section{Received: 10 December 2018 Accepted: 2 April 2019}

Published online: 08 May 2019

\section{References}

1. Afonso, C. L. et al. Taxonomy of the order Mononegavirales: update 2016 Arch. Virol. 161, 2351-2360 (2016).

2. Shi, T. et al. Global, regional, and national disease burden estimates of acute lower respiratory infections due to respiratory syncytial virus in young children in 2015: a systematic review and modelling study. Lancet $\mathbf{3 9 0}$, 946-958 (2017).

3. Paramore, L. C., Ciuryla, V., Ciesla, G. \& Liu, L. Economic impact of respiratory syncytial virus-related illness in the US: an analysis of national databases. Pharmacoeconomics 22, 275-284 (2004).

4. Shahabi, A., Peneva, D., Incerti, D., McLaurin, K. \& Stevens, W. Assessing variation in the cost of palivizumab for respiratory syncytial virus prevention in preterm infants. Pharm. Open 2, 53-61 (2018).

5. The IMpact-RSV Study Group. Palivizumab, a humanized respiratory syncytial virus monoclonal antibody, reduces hospitalization from respiratory syncytial virus infection in high-risk infants. Pediatrics 102, 531-537 (1998) 
6. Teng, M. N., Whitehead, S. S. \& Collins, P. L. Contribution of the respiratory syncytial virus $\mathrm{G}$ glycoprotein and its secreted and membrane-bound forms to virus replication in vitro and in vivo. Virology 289, 283-296 (2001).

7. Karron, R. A. et al. Respiratory syncytial virus (RSV) SH and G proteins are not essential for viral replication in vitro: clinical evaluation and molecular characterization of a cold-passaged, attenuated RSV subgroup B mutant. Proc. Natl. Acad. Sci. USA 94, 13961-13966 (1997)

8. Techaarpornkul, S., Barretto, N. \& Peeples, M. E. Functional analysis of recombinant respiratory syncytial virus deletion mutants lacking the small hydrophobic and/or attachment glycoprotein gene. J. Virol. 75, 6825-6834 (2001).

9. McLellan, J. S. et al. Structure of RSV fusion glycoprotein trimer bound to a prefusion-specific neutralizing antibody. Science 340, 1113-1117 (2013).

10. McLellan, J. S., Yang, Y., Graham, B. S. \& Kwong, P. D. Structure of respiratory syncytial virus fusion glycoprotein in the postfusion conformation reveals preservation of neutralizing epitopes. J. Virol. 85, 7788-7796 (2011).

11. Swanson, K. A. et al. Structural basis for immunization with postfusion respiratory syncytial virus fusion $\mathrm{F}$ glycoprotein (RSV F) to elicit high neutralizing antibody titers. Proc. Natl. Acad. Sci. USA 108, 9619-9624 (2011).

12. Bruhn, F. W. \& Yeager, A. S. Respiratory syncytial virus in early infancy. Circulating antibody and the severity of infection. Am. J. Dis. Child 131, 145-148 (1977).

13. Groothuis, J. R., Simoes, E. A. \& Hemming, V. G. Respiratory syncytial virus (RSV) infection in preterm infants and the protective effects of RSV immune globulin (RSVIG). Respiratory Syncytial Virus Immune Globulin Study Group. Pediatrics 95, 463-467 (1995)

14. Orvell, C., Norrby, E. \& Mufson, M. A. Preparation and characterization of monoclonal antibodies directed against five structural components of human respiratory syncytial virus subgroup B. J. Gen. Virol. 68(Pt 12), 3125-3135 (1987).

15. Lopez, J. A. et al. Antigenic structure of human respiratory syncytial virus fusion glycoprotein. J. Virol. 72, 6922-6928 (1998).

16. Garcia-Barreno, B. et al. Marked differences in the antigenic structure of human respiratory syncytial virus $\mathrm{F}$ and $\mathrm{G}$ glycoproteins. J. Virol. 63, 925-932 (1989).

17. Arbiza, J. et al. Characterization of two antigenic sites recognized by neutralizing monoclonal antibodies directed against the fusion glycoprotein of human respiratory syncytial virus. J. Gen. Virol. 73(Pt 9), 2225-2234 (1992).

18. McLellan, J. S. Neutralizing epitopes on the respiratory syncytial virus fusion glycoprotein. Curr. Opin. Virol. 11, 70-75 (2015).

19. Beeler, J. A. \& van Wyke Coelingh, K. Neutralization epitopes of the F glycoprotein of respiratory syncytial virus: effect of mutation upon fusion function. J. Virol. 63, 2941-2950 (1989).

20. Johnson, S. et al. Development of a humanized monoclonal antibody (MEDI493 ) with potent in vitro and in vivo activity against respiratory syncytial virus. J. Infect. Dis. 176, 1215-1224 (1997).

21. Lopez, J. A. et al. Conformational constraints of conserved neutralizing epitopes from a major antigenic area of human respiratory syncytial virus fusion glycoprotein. J. Gen. Virol. 74(Pt 12), 2567-2577 (1993).

22. McLellan, J. S. et al. Structural basis of respiratory syncytial virus neutralization by motavizumab. Nat. Struct. Mol. Biol. 17, 248-250 (2010).

23. Gilman, M. S. et al. Rapid profiling of RSV antibody repertoires from the memory B cells of naturally infected adult donors. Sci. Immunol. 1, aaj1879 (2016).

24. Kwakkenbos, M. J. et al. Generation of stable monoclonal antibody-producing $\mathrm{B}$ cell receptor-positive human memory B cells by genetic programming. Nat. Med 16, 123-128 (2010)

25. Ngwuta, J. O. et al. Prefusion F-specific antibodies determine the magnitude of RSV neutralizing activity in human sera. Sci. Transl. Med. 7, 309ra162 (2015).

26. Mousa, J. J., Kose, N., Matta, P., Gilchuk, P. \& Crowe, J. E. Jr. A novel prefusion conformation-specific neutralizing epitope on the respiratory syncytial virus fusion protein. Nat. Microbiol. 2, 16271 (2017).

27. Begona Ruiz-Arguello, M. et al. Effect of proteolytic processing at two distinct sites on shape and aggregation of an anchorless fusion protein of human respiratory syncytial virus and fate of the intervening segment. Virology 298, 317-326 (2002).

28. Gonzalez-Reyes, L. et al. Cleavage of the human respiratory syncytial virus fusion protein at two distinct sites is required for activation of membrane fusion. Proc. Natl. Acad. Sci. USA 98, 9859-9864 (2001).

29. Bolt, G., Pedersen, L. O. \& Birkeslund, H. H. Cleavage of the respiratory syncytial virus fusion protein is required for its surface expression: role of furin. Virus Res. 68, 25-33 (2000).

30. Scheid, A. \& Choppin, P. W. Two disulfide-linked polypeptide chains constitute the active F protein of paramyxoviruses. Virology 80, 54-66 (1977).

31. Schlender, J., Zimmer, G., Herrler, G. \& Conzelmann, K. K. Respiratory syncytial virus (RSV) fusion protein subunit F2, not attachment protein G, determines the specificity of RSV infection. J. Virol. 77, 4609-4616 (2003).
32. Lawlor, H. A., Schickli, J. H. \& Tang, R. S. A single amino acid in the F2 subunit of respiratory syncytial virus fusion protein alters growth and fusogenicity. J. Gen. Virol. 94, 2627-2635 (2013).

33. Krarup, A. et al. A highly stable prefusion RSV F vaccine derived from structural analysis of the fusion mechanism. Nat. Commun. 6, 8143 (2015).

34. Bermingham, I. M., Chappell, K. J., Watterson, D. \& Young, P. R. The Heptad Repeat $\mathrm{C}$ domain of the respiratory syncytial virus fusion protein plays a key role in membrane fusion. J. Virol. 92, JVI.01323-17 (2018).

35. Hicks, S. N. et al. Five residues in the apical loop of the respiratory syncytial virus fusion protein F2 subunit are critical for its fusion activity. J. Virol. 92, JVI.00621-18 (2018).

36. Stewart-Jones, G. B. et al. A cysteine zipper stabilizes a pre-fusion F glycoprotein vaccine for respiratory syncytial virus. PLoS ONE 10, e0128779 (2015).

37. Wong, J. J., Paterson, R. G., Lamb, R. A. \& Jardetzky, T. S. Structure and stabilization of the Hendra virus F glycoprotein in its prefusion form. Proc. Natl. Acad. Sci. USA 113, 1056-1061 (2016).

38. Yin, H. S., Wen, X., Paterson, R. G., Lamb, R. A. \& Jardetzky, T. S. Structure of the parainfluenza virus $5 \mathrm{~F}$ protein in its metastable, prefusion conformation. Nature 439, 38-44 (2006)

39. McLellan, J. S. et al. Structure-based design of a fusion glycoprotein vaccine for respiratory syncytial virus. Science 342, 592-598 (2013).

40. Gilman, M. S. et al. Characterization of a prefusion-specific antibody that recognizes a quaternary, cleavage-dependent epitope on the RSV fusion glycoprotein. PLoS Pathog. 11, e1005035 (2015).

41. Abou-Hamdan, A., Belot, L., Albertini, A. \& Gaudin, Y. Monomeric intermediates formed by vesiculovirus glycoprotein during its low- $\mathrm{pH}$ induced structural transition. J. Mol. Biol. 430, 1685-1695 (2018).

42. Cho, K. J. et al. Insight into structural diversity of influenza virus haemagglutinin. J. Gen. Virol. 94, 1712-1722 (2013).

43. Raymond, D. D. et al. Influenza immunization elicits antibodies specific for an egg-adapted vaccine strain. Nat. Med. 22, 1465-1469 (2016).

44. Baquero, E. et al. Intermediate conformations during viral fusion glycoprotein structural transition. Curr. Opin. Virol. 3, 143-150 (2013).

45. Munro, J. B. et al. Conformational dynamics of single HIV-1 envelope trimers on the surface of native virions. Science 346, 759-763 (2014).

46. Liu, J., Bartesaghi, A., Borgnia, M. J., Sapiro, G. \& Subramaniam, S. Molecular architecture of native HIV-1 gp120 trimers. Nature 455, 109-113 (2008).

47. Guttman, M. et al. CD4-induced activation in a soluble HIV-1 Env trimer. Structure 22, 974-984 (2014).

48. Rutten, L. et al. A universal approach to optimize the folding and stability of prefusion-closed HIV-1 envelope trimers. Cell Rep. 23, 584-595 (2018).

49. Lee, J. H. et al. Antibodies to a conformational epitope on gp41 neutralize HIV-1 by destabilizing the Env spike. Nat. Commun. 6, 8167 (2015).

50. Ke, Z. et al. The morphology and assembly of respiratory syncytial virus revealed by cryo-electron tomography. Viruses 10, 446 (2018).

51. Liljeroos, L., Krzyzaniak, M. A., Helenius, A. \& Butcher, S. J. Architecture of respiratory syncytial virus revealed by electron cryotomography. Proc. Natl. Acad. Sci. USA 110, 11133-11138 (2013).

52. Shaikh, F. Y. et al. A critical phenylalanine residue in the respiratory syncytial virus fusion protein cytoplasmic tail mediates assembly of internal viral proteins into viral filaments and particles. Mbio 3, e00270-11 (2012).

53. Lee, J. et al. Molecular-level analysis of the serum antibody repertoire in young adults before and after seasonal influenza vaccination. Nat. Med. 22, 1456-1464 (2016).

54. Paes, C. et al. Atomic-level mapping of antibody epitopes on a GPCR. J. Am Chem. Soc. 131, 6952-6954 (2009).

55. Morin, A. et al. Collaboration gets the most out of software. Elife 2, e01456 (2013).

56. Collaborative Computational Project, N. The CCP4 suite: programs for protein crystallography. Acta Crystallogr. D Biol. Crystallogr. 50, 760-763 (1994).

57. Potterton, E., Briggs, P., Turkenburg, M. \& Dodson, E. A graphical user interface to the CCP4 program suite. Acta Crystallogr. D Biol. Crystallogr. 59, 1131-1137 (2003)

58. Battye, T. G., Kontogiannis, L., Johnson, O., Powell, H. R. \& Leslie, A. G. iMOSFLM: a new graphical interface for diffraction-image processing with MOSFLM. Acta Crystallogr. D Biol. Crystallogr. 67, 271-281 (2011).

59. Evans, P. R. \& Murshudov, G. N. How good are my data and what is the resolution? Acta Crystallogr. D Biol. Crystallogr. 69, 1204-1214 (2013).

60. McCoy, A. J. et al. Phaser crystallographic software. J. Appl. Crystallogr. 40, 658-674 (2007)

61. Adams, P. D. et al. PHENIX: building new software for automated crystallographic structure determination. Acta Crystallogr. D Biol. Crystallogr. 58, 1948-1954 (2002).

62. Emsley, P. \& Cowtan, K. Coot: model-building tools for molecular graphics. Acta Crystallogr. D Biol. Crystallogr. 60, 2126-2132 (2004).

63. Grant, T., Rohou, A. \& Grigorieff, N. cisTEM, user-friendly software for single-particle image processing. Elife 7, eLife.35383 (2018). 


\section{Acknowledgements}

We thank Emilie Shipman, Daniel Wrapp, and John Ludes-Meyers in the McLellan laboratory for assistance with protein production and purification, Harrison Jones for assistance with flow cytometry, and members of the McLellan laboratory for helpfu comments on the manuscript. We also thank Calmune for monoclonal antibody isolation, Jennifer Lum for cloning, Gerrard Perdok for antibody production, Richard Voorzaat for performing binding assays, and the 19-ID beamline staff at the Structural Biology Center for assistance with collecting X-ray diffraction data. Results shown in this report are derived in part from work performed at Argonne National Laboratory, Structural Biology Center at the Advanced Photon Source. Argonne is operated by UChicago Argonne, LLC, for the U.S. Department of Energy, Office of Biological and Environmental Research under contract DE-AC02-06CH11357. We acknowledge the University of Texas College of Natural Sciences and award RR160023 of the Cancer Prevention and Research Institute of Texas for support of the EM facility at the University of Texas at Austin. Support for this work was provided by the National Institute of General Medical Sciences of the NIH awards T32GM008704 (M.S.A.G.) and Janssen.

\section{Author contributions}

Conceptualization, M.S.A.G., G.P., J.P.M.L., J.S.M.; Investigation, M.S.A.G., G.P., P.F.-H., A.vW.; Writing—original draft, M.S.A.G., J.P.M.L., and J.S.M.; Writing-reviewing and editing, M.S.A.G., A.vW., J.P.M.L., and J.S.M.; Visualization, M.S.A.G.; Supervision, J.S.M.

\section{Additional information}

Supplementary Information accompanies this paper at https://doi.org/10.1038/s41467019-09807-5.

Competing interests: J.S.M. is a named inventor on patents for vaccines and monoclonal antibodies for RSV, has received research funding from MedImmune and Janssen, has been a paid consultant for MedImmune, and is on the scientific advisory board for Calder Biosciences. M.S.A.G. is a named inventor on a patent application for RSV Fdirected single-domain antibodies. P.F.-H., G.P., A.vW., and J.P.M.L. are employees of Janssen, pharmaceutical companies of Johnson \& Johnson.

Reprints and permission information is available online at http://npg.nature.com/ reprintsandpermissions/

Journal peer review information: Nature Communications thanks the anonymous reviewers for their contribution to the peer review of this work.

Publisher's note: Springer Nature remains neutral with regard to jurisdictional claims in published maps and institutional affiliations.

cc (i) Open Access This article is licensed under a Creative Commons Attribution 4.0 International License, which permits use, sharing, adaptation, distribution and reproduction in any medium or format, as long as you give appropriate credit to the original author(s) and the source, provide a link to the Creative Commons license, and indicate if changes were made. The images or other third party material in this article are included in the article's Creative Commons license, unless indicated otherwise in a credit line to the material. If material is not included in the article's Creative Commons license and your intended use is not permitted by statutory regulation or exceeds the permitted use, you will need to obtain permission directly from the copyright holder. To view a copy of this license, visit http://creativecommons.org/ licenses/by/4.0/.

(c) The Author(s) 2019 